\title{
Cirurgia torácica geral em pacientes com revascularização miocárdica prévia
}

\author{
Paulo M. PEG O-FERNANDES * Fábio B. JATENE *, Alexandre WADA **, André Toraso \\ YAMAZAKI ${ }^{* *}$, Erika MIYOSHI ${ }^{\star *}$, Mário TERRA FILHO*, Adib D. JÁTENE *
}

RBCCV $44205-271$

\begin{abstract}
PEGGO-FERNANDES, P. M.; JATENE, F. B.; WADA, A.; YAMAZAKI, A. T.; MIYOSHI, E.; TERRA FILHO, M.; JATENE, A. D. - Cirurgia torácica geral em pacientes com revascularizaçāo miocárdica prévia. Rev. Bras. Cir. Cardiovasc., 10 (3): 144-149, 1995.

AESUMO: É analisada a experiéncia da Disciplina de Cirurgia Torácica da Faculdade de Medicina da Universidade de Sāo Paulo, em 24 pacientes submetidos a cirurgia torácica com revascularizaçăo miocárdica prévia. Estudaram-se variáveis em termos de morbi-mortalidade deste grupo de doentes. Os resultados mostraram que o melhor prognóstico foi encontrado nos pacientes submetidos a revascularizaçâo entre seis meses e cinco anos antes da cirurgia torácica. Notou-se, ainda, que os pacientes com infarto agudo do miocárdio apresentam um prognóstico tardio pior. O porte da cirurgia torácica e idade maior do que 70 anos foram fatores de risco tanto imediatos, quanto tardios.
\end{abstract}

DESCRITORES:Cirurgia torácica geral, em pacientes com revascularizaçăo prévia, fatores de risco.

\section{INTRODUÇĀO}

A insuficiência coronariana, em seus diversos graus, é afecção importante e causadora de complicaçōes em paciente cirúrgico năo cardiaco 3,4 . Diante deste fato, deve-se decidir pela viabilidade do procedimento cirúrgico em questăo, ou a melhoria da perfusão miocárdica previamnte à cirurgia. Atualmente, a maioria dos autores preconiza a revascularizaçăo do miocárdio previamente à cirurgia não cardíaca, como forma de estabilizar as condiçōes de perfusāo tecidual, tornando maiores as reservas coronarianas e miocárdicas, necessárias durante e após qualquer procedimento cirủrgico $2,6,7$. No entanto, pouco tem sido discutido quanto ao período após a revascularização, em que as condições do paciente seriam ideais para um novo ato cirúrgico ${ }^{7}$. Sabe-se que a evoluçāo da doença coronariana não estaciona após a revascularização. Portanto, haveria maior chance de insuficiência coronariana em outros locais, à medida em que decorre o tempo após a revascularização $e$, teoricamente, o prognóstico seria pior em paciente revascularizado há longo tempo antes da cirurgia atual. Por outro lado, a proximidade da cirurgia năo cardíaca com a revascularização prévia poderia levar a quadros de instabilidade coronariana, devido a efeitos de adaptaçăo a um regime de débito muito alto e à descompensação decorrente do ato cirúrgico.

O presente estudo visa analisar o tempo decorrido entre a revascularizaçăo miocárdica e a cirurgia torácica não cardiaca, correlacionando-o com pos-

Trabalho realizado na Disciplina de Cirurgia Torácica e Cardiovascular da Faculdade de Medicina da Universidade de Săo Paulo, Säo Paulo, SP, Brasil.

Recebido para publicaçăo em setembro de 1995.

- Do Instituto do Coraçăo do Hospital das Clínicas da Faculdade de Medicina da Universidade de Săo Paulo.

* Académicos da Faculdade de Medicina da Universidade de Säo Paulo.

Endereço para corresponde̊ncia: Paulo Pẻgo-Fernandes. Av. Dr. Enéas de Carvalho Aguiar, 44. Divisăo Cirúrgica. CEP: 05403-000 São Paulo, SP. Brasil. 
siveis complicaçōes intra e pós-operatórias. A cirurgia torácica, neste caso, poderia ser considerada como o protótipo do maior trauma cirúrgico a que um paciente poderia submeter-se. Concomitantemente, serăo analisados os riscos associados e as complicaçōes mais freqũentes.

\section{CASUISTICA E MÉTODOS}

Foram estudados, através de análise retrospectiva, 24 pacientes previamente revascularizados submetidos a procedimento cirúrgico torácico no InCór, no período de janeiro de 1987 a julho de 1995. Vinte e três pacientes eram do sexo masculino e 1 do sexo feminino. As idades variaram de 54 anos a 78 anos, com média de 64,1 anos.

Os pacientes foram divididos em grupos, segundo o tempo decorrido entre a revascularizaçāo prévia e a cirurgia torácica atual (Tabela 1).

TABELA 1

INTERVALO ENTRE REVASCULARIZACÃO MIOCÁRDICA E CIRURGIA TORÁCICA

\begin{tabular}{ll}
\hline TEMPO (MESES) & $N(\%)$ \\
\hline $0-6$ & $7(29,2)$ \\
$7-12$ & $2(8,3)$ \\
$13-59$ & $6(25,0)$ \\
$>60$ & $8(33,3)$ \\
DESCONHECIDO & $1(4,2)$ \\
\hline
\end{tabular}

Tempo decorrido entre a revascularização miocárdica prévia e a cirurgia torácica atual.

As cirurgias torácicas consistiram de 11 lobectomias, três ressecçōes de massas mediastinais, duas decorticaçōes (uma delas por videotoracoscopia (VTC), quatro biopsias pulmonares (uma por VTC), uma pneumectomia, uma talcagem, uma retirada de corpo estranho em espaço pleural e uma retirada de corpo estranho em hemitórax direito (HTD). As lobectomias e a pneumectomia foram agrupadas como cirurgias de grande porte e o restante em cirurgias outras.
As cirurgias prévias consistiram de 24 revascularizaçōes do miocárdio, sendo que 2 pacientes foram submetidos a troca de valva mitral, 1 a aneurismectomia de ventriculo esquerdo (VE), 3 a biopsia pulmonar e 1 a angioplastia, além de revascularização, concomitantemente.

Seis dos pacientes tinham infarto do miocárdio (IAM) prévio, 4 tinham insuficiência cardíaca congestiva (ICC), 7 eram sintomáticos para a insuficiência coronariana (ICO), 1 apresentava bloqueio atrioventricular total, 5 tinham hipertensăo arterial sistêmica (HAS), 2 tinham fibrilação atrial (FA) crônica, 1 miocardiopatia e 3 disfunçōes valvares.

\section{RESULTADOS}

Houve um óbito imediato, de paciente de 78 anos, que apresentava ICC. FA, miocardiopatia, insuficiência mitral e aórtica, e revascularização miocárdica prévia 11 anos antes da cirurgia, que consistiu de biopsia pleural com pleurodese por VTC, devido a adenocarcinoma. Este óbito ocorreu no $28^{\circ}$ dia de pós-operatório, por distúrbios hemodinåmicos, 20 dias após a alta hospitalar.

Seis pacientes apresentaram complicaçōes durante o período de internação hospitalar (1. hidropneumotórax; 1 , drenagem prolongada; 1 , descompensação da $1 \mathrm{CO} ; 2$, empiemas pleurais e 1 , broncopneumonia (BCP).

O tempo de internaçäo variou de um dia a 57 dias, com média de 14,2 dias.

Ocorreram seis óbitos tardios, um, insuficiência respiratória; dois, IAM, um, neoplasia e dois de causa desconhecida.

As complicaçōes e o bbito imediato foram agrupados como morbi-mortalidade.

Quanto à faixa etária, observamos uma morbimortalidade sensivelmente aumentada entre os pacientes na faixa de 70 anos a 79 anos $(60 \%)$. Ressalte-se o fato de que o único óbito imediato

TABELA 2

FAIXAETÁRIA

\begin{tabular}{ccccc}
\hline IDADE & $\boldsymbol{N}(\%)$ & MORBI-MORT. & OBITOS & INTERN. (DIAS) \\
\hline $50-59$ & $9(37,5)$ & $2(22,2)$ & $2(22,2)$ & 10,9 \\
$60-69$ & $10(41,7)$ & $1(10,0)$ & $3(39,0)$ & 16,7 \\
$70-79$ & $5(20,8)$ & $3(60,0)$ & $1(20,0)$ & 15,4 \\
TOTAL & $24(100,0)$ & $6(25,0)$ & $6(25,0)$ & 14,2 \\
\hline
\end{tabular}

Idade como fator de risco: Maior indice de morbi-mortalidade na faixa de $70-79$ anos; porém, a mortalidade tardia foi equivalente nas diversas faixas etárias. 
PEGO-FERNANDES, P. M.; JATENE, F. B.; WADA, A.; YAMAZAKI, A. T.; MIYOSHI, E.; TERRA FILHO, M.; JATENE, A. D. - Cirurgia torácica geral em pacientes com revascularizaçăo miocảrdica prévia. Rev. Bras. Cir. Cardiovasc., 10 (3): 144-149, 1995.

TABELA 3

SEXO

\begin{tabular}{ccccr}
\hline SEXO & $N(\%)$ & MORBI-MORT. & OBITOS & INTERN. (DIAS) \\
\hline Feminino & $1(4,2)$ & $0(0,0)$ & $1(100)$ & 9,0 \\
Masculino & $23(95,8)$ & $6(26,1)$ & $5(21,7)$ & 14,5 \\
\hline
\end{tabular}

Sexo como fator de risco: Predominância quase total do sexo masculino nesta amostra, impossibilitando a análise do sexo como fator de risco.

situou-se neste grupo. A mortalidade tardia, contudo, apresentou-se relativamente equilibrada, assim como o tempo de internação (Tabela 2).

O sexo dos pacientes foi predominantemente masculino $(95,8 \%)$, o que impossibilita qualquer tentativa de análise desta variável como fator de risco (Tabela 3 ).

Em relação ao intervalo entre a revascularizaçāo do miocárdio (RM) e a cirurgia atual (Tabela 4), notamos uma morbi-mortalidade maior entre os pacientes com cirurgia dentro dos seis primeiros meses após a RM $(42,9 \%)$, assim como um periodo de internação maior. Dentre os pacientes com intervalo superior a cinco anos, observamos uma mortalidade tardia olevada $(50,0 \%)$, sendo que a morbimortalidade referida trata-se do paciente que evoluiu com óbito imediato. Na faixa de um a cinco anos, ocorreu uma morbi-mortalidade e mortalidade intermediárias, e, na faixa de seis meses a um ano. não encontramos morbi-mortalidade $(0 \%)$, o mesmo na mortalidade tardia $(0 \%)$ e um tempo de internação reduzido (10 dias)

Entre os pacientes que tinham IAM prévio (Ta- bela 5), a morbi-mortalidade foi praticamente igual à dos que nāo tinham IAM prévio, porém, a mortalidade tardia foi três vezes maior, e o tempo de internação duas vezes maior.

Quanto à cirurgia torácica realizada, os pacientes com cirurgia maior (pneumectomias e lobectomias) apresentaram morbi-mortalidade, mortalidade tardia e tempo de internaçăo maiores do que os pacientes submetidos a cirurgia menor (Tabela 6).

Quando se faz a análise comparativa do intervalo entre a cirurgia atual e a RM, e a existência ou nāo de IAM prévio (Tabela 7), notamos que a maior morbi-mortalidade ocorre nos pacientes com intervalo menor do que um ano, que tinham IAM prévio, assim como o tempo de internaçāo foi três vezes maior do que a média dos outros grupos.

Dos pacientes com intervalo maior do que cinco anos, os que tinham IAM prévio näo apresentaram morbi-mortalidade. Entre os que nāo tinham IAM prévio, a morbi-mortalidade foi praticamente igual à média dos outros grupos (porém, tratava-se do único óbito imediato) e a mortalidade tardia foi de $75 \%$ entre os que sobreviveram neste grupo. Na faixa de

TABELA 4

INTERVALO RMPRE-CIR. TORÁCICA (IRMCIR)

\begin{tabular}{llcll}
\hline TEMPO (mês) & N $(\%)$ & MORBI-MORT. & OBITOS & INTERN. (DIAS) \\
\hline $0-6$ & $7(29,2)$ & $3(42,9)$ & $1(14,3)$ & 17,3 \\
$7-12$ & $2(8,3)$ & $0(0,0)$ & $0(0,0)$ & 10,0 \\
$13-60$ & $6(25,0)$ & $2(33,3)$ & $1(16,7)$ & 14,3 \\
$>60$ & $8(33,3)$ & $1(12,5)$ & $4(50,0)$ & 13,1 \\
DESCONHECIDO & $1(4,2)$ & $0(0,0)$ & $0(0,0)$ & 10,0 \\
\hline TOTAL & $24(100)$ & $6(25,0)$ & $6(25,0)$ & 14,2 \\
\hline
\end{tabular}

Intervalo entre a revascularizaçăo prévia e a cirurgia torácica.

TABELA 5

IAM (INFARTO AGUDO DO MIOCARDIO) PREVIO

\begin{tabular}{lrccc}
\hline & $N(\%)$ & MORBI-MORT. & OBITOS & INTERN. (DIAS) \\
\hline $\operatorname{sim}$ & $6(25,0)$ & $1(16,7)$ & $3(50,0)$ & 23,0 \\
Nāo & $18(75,0)$ & $5(27,8)$ & $3(16,7)$ & 11,3 \\
\hline
\end{tabular}

IAM prévio: Infarto agudo do miocárdio como fator de risco em revascularizados previamente. 
PÊGO-FERNANDES, P. M; JATENE, F.B; WADA, A.;YAMAZAKI, A. T; MIYOSHI, E;; TERRA FILHO, M.; JATENE, A. D. - CIrURgIa torácica geral em pacientes com revascularizaçăo miocárdica prévia. Rev. Bras. Cir. Cardiovasc., 10 (3): 144-149, 1995.

TABELA 6

CIRURGIA TORÁCICA

\begin{tabular}{llclr}
\hline & $N(\%)$ & MORBI-MORT. & OBITOS & INTERN. (DIAS) \\
\hline Lobec/Pneum & $12(50,0)$ & $4(33,3)$ & $4(33,3)$ & 19,1 \\
Outras & $12(50,0)$ & $2(16,7)$ & $2(16,7)$ & 9,4 \\
\hline TOTAL & $24(100,0)$ & $6(25,0)$ & $6(25,0)$ & 14,2 \\
\hline
\end{tabular}

Cirurgia torácica: Importância do porte da cirurgia torácica como fator de risco (lobectomia e pneumectomia: grande porte, outras biopsias, ressecçoes de massas mediastinals, decorticaçðes, talcagem e retirada de corpo estranho).

TABELA 7

IRMCIR/IAMPRÉ

\begin{tabular}{|c|c|c|c|c|c|}
\hline IRMCIR & $I A M$ & $N(\%)$ & MORBI-MORT. & ÓBITOS & INTERN. (DIAS) \\
\hline $0-12$ & $\begin{array}{l}\text { sim } \\
\text { nāo }\end{array}$ & $\begin{array}{l}2(22,2) \\
7(77,8)\end{array}$ & $\begin{array}{l}1(50,0) \\
2(28,6)\end{array}$ & $\begin{array}{l}1(50,0) \\
0(0,0)\end{array}$ & $\begin{array}{l}33,0 \\
10,7\end{array}$ \\
\hline $13-59$ & $\begin{array}{l}\text { sim } \\
\text { não }\end{array}$ & $\begin{array}{l}0(0,0) \\
6(100,0)\end{array}$ & $2(33,3)$ & $1(16,7)$ & 14,3 \\
\hline$>60$ & $\begin{array}{l}\text { sim } \\
\text { nào }\end{array}$ & $\begin{array}{l}4(50,0) \\
4(50,0)\end{array}$ & $\begin{array}{l}0(0,0) \\
1(25,0)\end{array}$ & $\begin{array}{l}2(50,0) \\
2(50,0)\end{array}$ & $\begin{array}{r}18,0 \\
8,2\end{array}$ \\
\hline DESCONHECIDO & $\begin{array}{l}\text { simm } \\
\text { náo }\end{array}$ & $\begin{array}{l}0(0,0) \\
1(100,0)\end{array}$ & $0(0,0)$ & $0(0,0)$ & 10,0 \\
\hline
\end{tabular}

IRMCIR: intervalo entre a revascularizaçăo miocárdica e a cirurgia torácica.

IAM PRE: infarto agudo do miocárdio prévio.

Análise bifatorial de intervalo entre a revascularizaçăo miocárdica e a cirurgia torácica (IRMCIR), e presença de infarto agudo do miocárdio (IAM) prévio.

um a cinco anos, os índices de morbi-mortalidade e mortalidade tardia foram os menores.

$\mathrm{Na}$ análise bi-fatorial entre intervalo da cirurgia torácica e RM prévios e porte da cirurgia (Tabela 8), notamos que a maior morbi-mortalidade ocorreu nos pacientes com intervalo menor que um ano submetidos a cirurgias de grande porte $(60 \%)$, e a maior mortalidade tardia ocorreu nos pacientes com intervalo maior do que cinco anos submetidos a cirurgias de grande porte. Novamente, nota-se uma situação de menor morbi-mortalidade no grupo com intervalo entre um e cinco anos, porém é evidente a influência do fator porte da cirurgia, principalmente na morbi-mortalidade, mas também na mortalidade tardia.

Quando se analisa o fator IAM prévio conjuntamente com o porte da cirurgia, observa-se menor morbi-mortalidade entre os pacientes que se submeteram a cirurgias menores, seja qual for sua situação em relação ao IAM prévio (Tabela 9). Também o tempo de internação foi maior entre os pacientes submetidos a procedimentos maiores. A mortalidade tardia foi maior entre os pacientes submetidos a procedimentos maiores, porém a presença de IAM prévio produziu os maiores indices de óbitos tardios.

\section{COMENTÁRIOS}

Com relaçăo ao intervalo entre cirurgia torácica atual e RM prévia, notamos uma morbi-mortalidade muito elevada na faixa de um a seis meses $(42,9 \%)$, com mortalidade tardia praticamente igual à medida dos outros grupos. Este fato pode ser decorrente da interposiçāo de complicaçōes das duas cirurgias, ou de um funcionamento ainda não ideal do bypass. Porém, esta afirmativa é apenas teórica, necessitando de confirmaçāo experimental.

Os pacientes com intervalo maior do que cinco anos entre a RM e a cirurgia atual apresentaram uma morbi-mortalidade relativamente baixa $(12,5 \%)$, porém, neste grupo, ocorreu o único b́bito imediato. Além disso, dos que sobreviveram, $57 \%$ evoluíram com óbitos tardios. A explicação parece ser o maior tempo de doença coronariana entre os pacientes com RM mais distante. Estes pacientes, além de apresentarem a doença por mais tempo, ainda tinham maior tempo de evoluçăo de outros locais de aterosclerose, com maior tempo de doença sem tratamento cirúrgico.

Com os dados obtidos, notamos um melhor prognóstico entre os pacientes submetidos a cirurgia no período de seis meses a cinco anos após a 
PÉGO-FERNANDES, P.M.; JATENE, F.B.; WADA, A.; YAMAZAKI, A. T.; MIYOSHI, E.; TERRA FILHO, M.; JATENE, A. D. - Clirurgia torácica geral em pacientes com revascularizaçăo miocárdica prévia. Rev. Bras. Cir. Cardiovasc., 10 (3): 144-149, 1995.

TABELA 8

IRMCIR/CIR. TORACICA

\begin{tabular}{|c|c|c|c|c|c|}
\hline IRMCIR & CIRURGIA & $N(\%)$ & MORBI-MORT. & бвITOS & INTERN. (DIAS) \\
\hline $0-12$ & $\begin{array}{l}\text { lob/pneu } \\
\text { outras }\end{array}$ & $\begin{array}{l}5(55,6) \\
4(44,4)\end{array}$ & $\begin{array}{l}3(60,0) \\
0(0,0)\end{array}$ & $\begin{array}{l}0(0,0) \\
1(25,0)\end{array}$ & $\begin{array}{r}20,6 \\
9,5\end{array}$ \\
\hline 13.59 & $\begin{array}{l}\text { lob/pneu } \\
\text { outras }\end{array}$ & $\begin{array}{l}2(33,3) \\
4(66,7)\end{array}$ & $\begin{array}{l}1(50,0) \\
1(25,0)\end{array}$ & $\begin{array}{l}1(50,0) \\
0(0,0)\end{array}$ & $\begin{array}{l}20,5 \\
11,3\end{array}$ \\
\hline$>60$ & $\begin{array}{l}\text { lob/pneu } \\
\text { outras }\end{array}$ & $\begin{array}{l}5(62,5) \\
3(37,5)\end{array}$ & $\begin{array}{l}0(0.0) \\
1(33,3)\end{array}$ & $\begin{array}{l}3(60,0) \\
1(33,3)\end{array}$ & $\begin{array}{r}17,0 \\
6,7\end{array}$ \\
\hline DESCONHECIDO & $\begin{array}{l}\text { lob/pneu } \\
\text { outras }\end{array}$ & $\begin{array}{l}0(0,0) \\
1(100,0)\end{array}$ & $0(0,0)$ & $0^{-}$ & $10, \overline{0}$ \\
\hline
\end{tabular}

lob: Iobectomia

pneu: pneumectomia

Análise bifatorial de IRMCIR e porte da cirurgia torácica.

TABELA 9

IAMPRE/CIR. TORACICA

\begin{tabular}{|c|c|c|c|c|c|}
\hline CIRURGIA & IAM Pré & $N(\%)$ & MORBJ-MORT. & סBITOS & INTERN. (DIAS) \\
\hline Lob/pneu & $\begin{array}{l}\text { sim } \\
\text { năo } \\
\text { subtotal }\end{array}$ & $\begin{array}{l}5(41,7) \\
7(58,3) \\
12(50,0)\end{array}$ & $\begin{array}{l}1(20,0) \\
3(42,9) \\
4(33,3)\end{array}$ & $\begin{array}{l}2(40,0) \\
2(28,6) \\
4(33,3)\end{array}$ & $\begin{array}{l}25,8 \\
14,3 \\
17,1\end{array}$ \\
\hline outras & $\begin{array}{l}\text { sim } \\
\text { não } \\
\text { subtotal }\end{array}$ & $\begin{array}{l}1(8,3) \\
11(91,7) \\
12(50,0)\end{array}$ & $\begin{array}{l}0(0,0) \\
2(18,2) \\
2(16,7)\end{array}$ & $\begin{array}{l}1(100,0) \\
1(9,1) \\
2(16,7)\end{array}$ & $\begin{array}{l}9,0 \\
9,5 \\
9,4\end{array}$ \\
\hline TOTAL & & $24(100,0)$ & $6(25,0)$ & $6(25,0)$ & 14,2 \\
\hline
\end{tabular}

Análise bifatorial de IAM prévio e porte da cirurgia torácica.

RM prévia. De fato, a morbi-mortalidade e a mortalidade tardia foram as menores neste grupo. Além disso, o grupo de operados após sete a 12 meses não apresentou nenhum caso de morbi-mortalidade ou de mortalidade tardia, e o tempo de internaçăo foi o segundo menor. Portanto, a faixa mais segura para se operar um paciente seria após sete meses a um ano após a RM prévia. Assim, eliminam-se as complicaçōes do ato cirúrgico prévio e trabalha-se com um paciente compensado a nivel de perfusão coronariana

A presença de IAM também mostrou ser um fator de risco, principalmente em relaçāo à mortalidade tardia. Este fato pode ser ligado diretamente à maior gravidade da cardiopatia, que, se bem compensada no intra-operatório e no pó-operatório, pode levar a índices muito baixos de complicaçōes imediatas 1. 5. Porém, a evolução da doença coronariana continua após a cirurgia torácica e o prognóstico tardio permanece mau.

O porte da cirurgia determinou um índice de morbi-mortalidade e mortalidade tardia alto. Em relaçāo à morbi-mortalidade, o maior trauma cirúrgico em um paciente com disfunção coronariana determinaria um prognóstico imediato pior, já que o paciente năo possui reserva coronariana suficiente para entrentar tamanho stress cirúrgico. A mortalidade tardia elevada poderia ser explicada pela maior gravidade da enfermidade torácica em pacientes submetidos a procedimentos maiores.

Sabemos que a idade acima de 70 anos é fator de risco para qualquer tipo de cirurgia 3, 4. Este estudo confirma o fato, já que se obteve uma morbimortalidade elevada nesta faixa etária. Outros fatores de risco, como cardiopatias associadas, gravidade da doença torácica e outras lesōes também săo importantes $1,3-5$, este estudo limitou-se a analisar os fatores relacionados a coronariopatia e ao tempo decorrido entre sua correção e a cirurgia proposta, devido ao número de pacientes não ser suficiente para subdivisāo em muitos grupos.

Estes dados mostram uma vantagem na realizaçăo da cirurgia torácica, nos pacientes que se submeteram a RM entre seis meses e cinco anos antes. Além disso, deve-se levar em consideraçăo idade, cardiopatias associadas, presença de IAM prévio e outras afecçōes, para se planejar o tipo de tratamento mais adequado e, quando cirúrgico, definir a sua extensāo. 
PÉGO-FERNANDES, P. M.; JATENE, F. B; WADA. A.; YAMAZAKI, A. T.; MIYOSHI, E.; TERRAFILHO, M.; JATENE, A. D. - Cirurgia torácica geral em pacientes com revascularizaçāo miocárdica prévia.

Rev. Bras. Cir. Cardiovasc., $10(3): 144-149,1995$

RBCCV $44205-271$

PÊGO-FERNANDES, P. M.; JATENE, F. B.; WADA, A.; YAMAZAKI, A. T.; MIYOSHI, E.; TERRA FILHO, M.; JATENE, A. D. - Non-cardiac thoracic surgery in patients with previous myocardial revascularization. Rev. Bras. Cir. Cardiovasc, 10 (3): 144-149, 1995.

ABSTRACT: It was analysed the experience of the Department of Thoracic Surgery of Faculdade de Medicina da Universidade de Săo Paulo, in 24 patients undergone non-cardiac thoracic surgeries, and who had been submitted to previous myocardial revascularization. Factors related to morbi-mortality were studied in this group of patients. Results showed a better outcome in patients who were submitted to myocardial revascularization between 6 months and 5 years before the thoracic surgery. It was noted a worst outcome in patients with previous myocardial infarction. The kind of surgeries and age greater than 70 years old showed to be important immediate and late risk factors.

DESCRIPTORS: Thoracic surgery, non-cardiac, in patients with previous revascularization, risk factors

\section{REFERÊNCIAS BIBLIOGRÁFICAS}

1 AULER Jr., J, O. C. - Anestesia para cardiopatas submetidos a cirurgia năo cardiaca. In: Socesp cardiologia: atualizaçáo e reciclagem. Săo Paulo, Atheneu, 1993.

2 FOSTER, E. D.; DAVIS, K. B.; CARPENTIER, J. A.; ABELLE, S.; FRAY, D. - Risk of noncardiac operation in patients with defined coronary disease: the Coronary Artery Surgery Study (CASS) registry experience. Ann. Thorac. Surg, 41: 43-49, 1986.

3 GOLDMAN, L.; CALDERA, D. L.; SOUTHWICK, F. S.; NUSSBAUN, S. R.; MURRAY, B.; O'MALLEY, T. A.; GOROLL, A. H.; CAPLAN, C. H.; NOLAN, J.; BURKE, D. S; KROGSTAD, D ; CARABELLO, B.; SLATER, E. E. - Cardiac risk factors and complications in noncardiac surgery. Medicine, 57: 357-370, 1978.

4 GOLDMAN, L.; CALDERA, D. L.; SOUTHWICK, F. S. NUSSBAUN, S. R.; MURRAY, B.; O'MALLEY, T. A.;
GOROLL, A. H.; CAPLAN, C. H.; NOLAN, J.; BURKE, D. S.; KROGSTAD, D.; CARABELLO, B.; SLATER, E. E. - Multifactorial index of cardiac risk in noncardiac surgical procedures. N. Engl. J. Med., 297: 845-850, 1977

5 GOLDMAN, L. \& BRAUNWALD, E. - General anesthesia and noncardiac surgery in patients with heart disease. In: BRAUNWALD, E. Heart disease: a textbook of cardiovascular medicine. 4. ed. Philadelphia, WB Saunders, 1992. p. 1708-1720.

6 MAHAR, L. J.; STEEN, P. A.; TINKER, J. H.; VLIETSTRA, R. E.; SMITH, H. C.; PLUTH, J. R. Perioperative myocardial infarction in patients with coronary artery disease with and without aortacoronary artery bypass grafts. $J$. Thorac. Cardiovasc. Surg., 76: 533-537, 1978.

7 MICHEL, L. A.; JAMART, J.; BRADPIECE, H. A.; MALT, R. A. - Prediction of risk in noncardiac operations after cardiac operations. J. Thorac. Cardiovasc. Surg., 100: 595-605, 1990. 